\title{
Geometrically Nonlinear Transversal Vibrations of Corrugated Cylindrical Shells
}

\author{
M. V. Marchuk*1,2, T. V. Goriachko' ${ }^{1}$ V. S. Pakosh ${ }^{1}$, O. F. Lesyk ${ }^{3}$ \\ ${ }^{1}$ Pidstryhach Institute for Applied Problems of Mechanics and Mathematics, NASU, L’viv, Ukraine \\ ${ }^{2}$ National University «L'viv Polytechnic», L'viv, Ukraine \\ ${ }^{3}$ Ternopil' National Economic University, MESU, Ternopil', Ukraine \\ *Corresponding author. E-mail: mv_marchuk@ukr.net
}

Paper received 10.12.18; Accepted for publication 17.12.18.

\section{https://doi.org/10.31174/SEND-NT2018-186VI22-16}

\begin{abstract}
The expressions for the geometric characteristics of the median surface of the corrugated cylindrical shell, depending on the frequency and amplitude of the corrugation, are obtained. The character of the dependence of the first natural frequency of geometrically nonlinear vibrations of corrugated elongated cylindrical panels on the corrugation frequency is investigated.
\end{abstract}

Keywords: corrugated cylindrical shell, vibrations, natural frequencies, geometrically nonlinearity.

Introduction. Shell elements are one of the most common components of loaded structures and structures of various intended use. This is due to their rational material capacity and the ability to provide the necessary stiffness in certain areas that require operating conditions. Among the various types of shells, a special place is occupied by circular cylindrical shells and their fragments. A large number of works [10] is devoted to the simulation of deformation and the methods of their calculation for the actions of both static and dynamic loads. Less investigated are cylindrical shells coiled in the direction of the circular coordinates, especially for dynamic geometrically nonlinear deformation, in particular their transverse oscillations. To avoid resonance phenomena due to the effects of intense vibration loads, it is necessary at the design stage to determine the spectra of the natural frequencies of the specified structural elements.

Linear free vibrations of corrugations in the direction of the angular coordinates of cylindrical shells were investigated in [3, 7-9]. In [1, 4, 11] their geometrically nonlinear vibrations were investigated. Various applied shell theories were used for this purpose. However, such an approach does not allow fully (exactly) take into account the geometry of the middle sections of such shells and the specificity of the elastic characteristics of materials used for their manufacture. In order to eliminate this shortcoming in this paper, a method is presented for determining the amplitude-frequency characteristics of corrugated cylindrical shells on the basis of spatial dynamic geometrically nonlinear relations of the theory of elasticity.

Problem statement. At first, consider the thin curvilinear elastic layer in thickness $h$ with a cylindrical median surface of the radius $R$. We refer this surface to the natural mixed coordinate system $\alpha_{1}=\varphi, \alpha_{2}=z, \alpha_{3}=r$ (see Fig. 1).

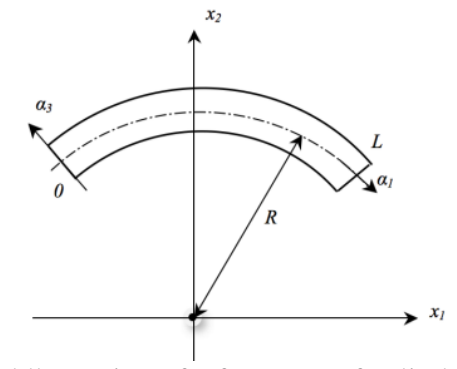

Fig. 1. The middle section of a fragment of cylindrical circular shell

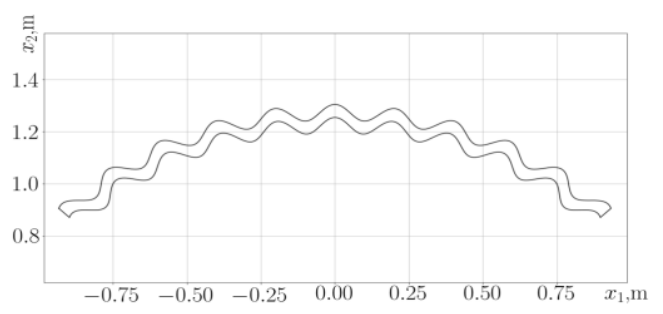

Fig. 2. The middle section of a fragment of corrugated cylindrical circular shell

Now let the mid-section of the layer have the form, as in Figure 2. We introduce on its mid-surface the same as for a circular cylindrical surface, a mixed coordinate system $\alpha_{i}, i=1,2,3$, which is connected with Cartesian $x_{i}, i=1,2,3$ by relations

$$
\begin{gathered}
x_{1}=\left(R+\alpha_{3}+g_{A} \cos \left(g_{v} a\left(\alpha_{1}\right)\right)\right) \cos \left(a\left(\alpha_{1}\right)\right) ; \\
x_{2}=\left(R+\alpha_{3}+g_{A} \cos \left(g_{v} a\left(\alpha_{1}\right)\right)\right) \sin a\left(\alpha_{1}\right) ; x_{3}=\alpha_{2}(1)
\end{gathered}
$$

where $R$-the radius of the median surface of the circular cylindrical shell, the upper and lower facial surfaces of which pass through the vertices of the corrugations; $L=\alpha_{1}^{0}-$ the length of the arc of the creature; $g_{A}-$ amplitude of corrugation; $g_{v}-$ corrugation frequency; $a\left(\alpha_{1}\right)=\pi / 2+K\left(L / 2-\alpha_{1}\right) ; K=1 / R$.

Functions that determine the characteristics of a geometrically nonlinear vibration process describe the dependencies [6]:

1. motion equations

$$
\operatorname{div} \hat{S}=\rho \frac{\partial^{2} U}{\partial t^{2}}
$$

2. elasticity relations

$$
\hat{\Sigma}=\tilde{A} \otimes \hat{\varepsilon}(3)
$$

3. deformation relation between the strain tensor components $\hat{\varepsilon}$ and the components of the elastic displacement vector $\vec{U}=u_{i} \vec{e}_{i} \vec{e}_{j}$

$$
\varepsilon_{i j}=\frac{1}{2}\left(\nabla_{i} u_{j}+\nabla_{j} u_{i}+\nabla_{i} u^{k} \nabla_{j} u_{k}\right)
$$

4. relation between the components $S^{i j}$ of the nonsymmetrical Kirchhoff stress tensor $\hat{S}$ and the components $\sigma^{i k}$ of the symmetric Piola stress tensor $\hat{\Sigma}$

$$
S^{i j}=\sum_{k} \sigma^{i k}\left(\delta_{k}^{j}+\nabla_{k} u^{j}\right)(5)
$$


In equations (2) and (3) $\tilde{A}$ - tensor of elastic characteristics of the layer, and $\rho$ - its density.

Boundary conditions on the front surfaces of the shell $\alpha_{3}= \pm h / 2$ for the free vibrations has the form

$$
S^{3 i}\left(\alpha_{1}, \alpha_{2}, \pm h / 2, t\right)=0,\left|\alpha_{i}\right| \leq \alpha_{i}^{0}, i=1,2 \text { (6) }
$$

and on its ends $\alpha_{1}= \pm \alpha_{1}^{0}$ at their hinged fixing on the lower front surface $\alpha_{3}=-h / 2$ :

$$
\begin{gathered}
S^{1 i}\left( \pm \alpha_{1}^{0}, \alpha_{2}, \alpha_{3}, t\right)=0,(7) \\
u_{i}\left( \pm \alpha_{1}^{0}, \alpha_{2},-h / 2, t\right)=0, i=1,2,3, a=0, l(8)
\end{gathered}
$$

The motion equations (2) together with relations (3) (5) and boundary conditions (6)-(8) are describe geometrically nonlinear transverse vibrations of the corrugated elastic layer.

Solution of the problem. For the radius vector of shell generatrix from (1) we have the expression

$$
\bar{r}\left(\alpha_{1}\right)=\left(\begin{array}{l}
\left(R+g_{A} \cos \left(g_{v} a\left(\alpha_{1}\right)\right)\right) \cos \left(a\left(\alpha_{1}\right)\right) \\
\left(R+g_{A} \cos \left(g_{v} a\left(\alpha_{1}\right)\right)\right) \sin \left(a\left(\alpha_{1}\right)\right)
\end{array}\right) \text { (9) }
$$

Radius vector of each point of the median cross-section of the layer

$$
\bar{R}=\bar{r}\left(\alpha_{1}\right)+\alpha_{3} \bar{n}\left(\alpha_{1}\right) .
$$

For vectors of the covariant base of the median surface of the corrugated layer (corrugated cylindrical shell) from (1) together with (9) we obtain:

$$
\begin{gathered}
R_{1}=(w \sin (a)+z \cos (a)) \vec{e}_{1}+(-w \cos (a)+z \sin (a)) \vec{e}_{2} ; \\
R_{2}=\cos (a) \vec{e}_{1}+\sin (a) \vec{e}_{2} ; R_{3}=\vec{e}_{3},
\end{gathered}
$$

where $\quad w=w\left(\alpha_{1}, \alpha_{2}\right)=q+g_{A} K \cos \left(g_{v} a\right)$ $z=z\left(\alpha_{1}\right)=g_{A} g_{v} K \sin \left(g_{v} a\right) ; \vec{e}_{i}, i=\overline{1,3}-$ the basic vectors of the Cartesian system $x_{i}, i=\overline{1,3}$.

For radius vector of the tangent to the generatrix we obtained the following expression

$$
\bar{r}^{\prime}\left(\alpha_{1}\right)=\left(\begin{array}{c}
\left(1+g_{A} K \cos \left(g_{v} a\right)\right) \sin (a)+g_{A} g_{v} K \sin \left(g_{v} a\right) \cos (a) \\
-\left(1+g_{A} K \cos \left(g_{v} a\right)\right) \cos (a)+g_{A} g_{v} K \sin \left(g_{v} a\right) \sin (a)
\end{array}\right) .
$$

Then for the normal to the generatrix we gives

$$
\begin{gathered}
\bar{n}\left(\alpha_{1}\right)=\frac{1}{\sqrt{\bar{r}^{\prime}\left(\alpha_{1}\right)_{x}^{2}+\bar{r}^{\prime}\left(\alpha_{1}\right)_{y}^{2}}}\left(\begin{array}{c}
-\bar{r}^{\prime}\left(\alpha_{1}\right)_{y} \\
\bar{r}^{\prime}\left(\alpha_{1}\right)_{x}
\end{array}\right)=\frac{1}{\sqrt{\left(1+g_{A} K \cos \left(g_{v} a\right)\right)^{2}+\left(g_{A} g_{v} K \sin \left(g_{v} a\right)\right)^{2}}} \times \\
\times\left(\begin{array}{l}
\left(1+g_{A} K \cos \left(g_{v} a\right)\right) \cos (a)-g_{A} g_{v} K \sin \left(g_{v} a\right) \sin (a) \\
\left(1+g_{A} K \cos \left(g_{v} a\right)\right) \sin (a)+g_{A} g_{v} K \sin \left(g_{v} a\right) \cos (a)
\end{array}\right) .
\end{gathered}
$$

In Figures 3 and 4 depicts the location of vectors normal to the median surface of the corrugated cylindrical shell for two different corrugation frequencies.

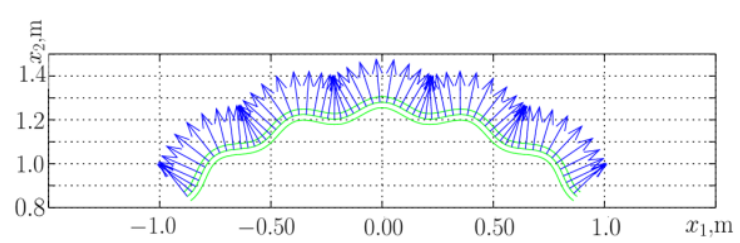

Fig. 3. Appearance of the section of the corrugated shell and normal to the median surface at $L=1 \mathrm{~m}, R=1.25 \mathrm{~m}, h=0.05 \mathrm{~m}$,

$$
g_{A}=0.03 \mathrm{~m}, g_{v}=20
$$

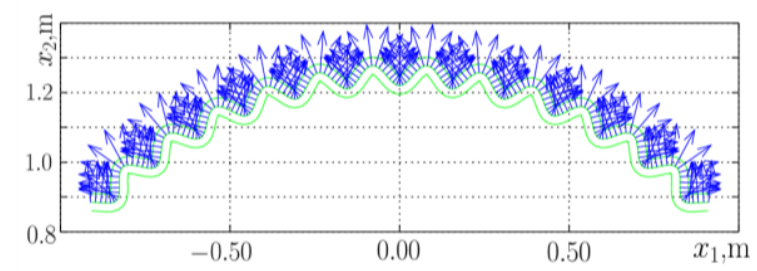

Fig. 4. Appearance of the section of the corrugated shell and normal to the median surface at $L=1 \mathrm{~m}, R=1.25 \mathrm{~m}, h=0.05$ $\mathrm{m}, g_{A}=0.03 \mathrm{~m}, g_{v}=50$

Similarly, we construct a covariant base and normal and tangent on the facial surfaces of the considered shell. This is necessary for the possibility of using the proposed and substantiated numerical method in [6] to find a finite number of natural frequencies and modes of elongated cylindrical shells with arbitrary form of generators in geometrically nonlinear vibrations. The method is based on the linear approximation of displacements of the shell points along the normal coordinate, in combination with the finite element method, according to the tangential coordinate on the median surface shell [2]. The resulting system of nonlinear algebraic equations with respect to approximation coefficients is solved by an improved perturbation method, which was proposed and theoretically substantiated in [5].

Numerical example and conclusions. As an object, an elongated cylindrical shell with a length of generatrix $2 L=2 \mathrm{~m}$, the radius of the median surface of the shell, whose facial surfaces pass through the edges of the corrugations, $R=1.25 \mathrm{~m}$ and elastic characteristics, are chosen: $E=2.1 \cdot 10^{11} \mathrm{H} / \mathrm{M}^{2}, v=0.3, G=8.1 \cdot 10^{10} \mathrm{H} / \mathrm{M}^{2}$ and density $\rho=8 \cdot 10^{3} \mathrm{~kg} / \mathrm{m}^{3}$. The influence of corrugation frequency $g_{v}$ on the minimum natural frequency $\omega_{\min }$ is investigated.

Table. The dependence of the $\omega_{\min }$ on the $g_{v}$

\begin{tabular}{|c|c|c|c|c|c|c|c|c|c|c|c|c|}
\hline$g_{v}$ & 2 & 4 & 6 & 8 & 10 & 20 & 50 & 80 & 100 & 200 & 300 & 500 \\
\hline$\omega_{\min }[\mathrm{Hz}]$ & 799 & 775 & 692 & 1025 & 1056 & 3658 & 6383 & 6936 & 7709 & 5559 & 4914 & 3195 \\
\hline
\end{tabular}

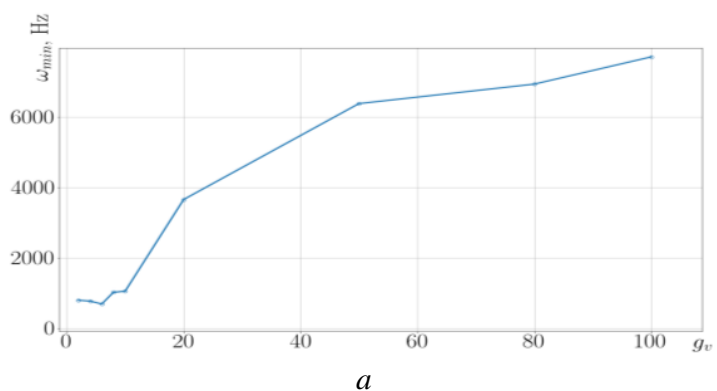

Fig 5. The dependence of the minimum natural frequency $\omega_{\min }$ on the corrugation frequency $g_{v}$

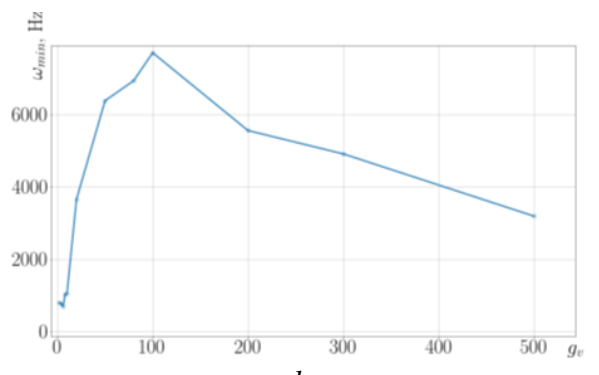


As can be seen from the results shown in the Table and graphs in Fig. 5 the minimum $\omega_{\text {min }}$ is achieved at $g_{v}=6$, which coinciding with the conclusions of article [7]. Also from Figure $5 \mathrm{~b}$ it can be concluded that when $g_{v} \rightarrow \infty$ the value $\omega_{\min }$ goes to a certain value greater than the first proper frequency of the non-circular cylindrical shells. This is due to the fact that the boundary transition obtained by the smooth shell has a greater thickness than the original non-enveloped shell.

\section{REFERENCES}

1. Ahmed Mousa Khalifa, Solving the Vibration Problem of Inhomogeneous Orthotropic Cylindrical Shells with HoopCorrugated Oval Cross Section, Comptes Rendus Mécanique, 343, No.9 (2015) $482-494$. Doi: 10.1016/j.crme.2015.07.007.

2. Bathe Klaus-Jurgen, Finite Element Procedures, 2nd Ed., Watertown, MA 2014. - 1063p.

3. G.R. Gulgazaryan, L.G. Gulgazaryan, Vibrations of a corrugated orthotropic cylindrical shell with free edges, Int Appl. Mech., 42, Issue 12, 1398 - 1413 (2006).

4. Kim Young-Wann, Vibration Analysis of Longitudinally Corrugated Cylindrical Shells, Transactions of the Korean Soc for Noise and Vibration Eng., 26 (2016) 851 - 856.

5. Lewandowski R. Free Vibration of Structures with Cubic Non-linearity-remarks on Amplitude Equation and Rayleigh Quotient // Comput. Methods Appl. Mech. Eng. - 2003. 192(13). - P. 1681-1709.

6. M. Marchuk, T. Goriachko, V. Pakosh, Geometrically Nonlinear Free Transversal Vibrations of Thin-Walled Elongated Panels with Arbitrary Generatrix, Vibrations in Physical Systems, 26 (2014) $153-160$

7. S.V. Puzyrev, O svobodnykh kolebaniyakh nekrugovykh tsilindricheskikh obolochek $\mathrm{s}$ gofrirovannym ellipticheskim secheniyem, Zb. nauk. prats' Nats. un-tu korablebuduvannya, 1, (2013) $47-53$.

8. S.V. Puzyrev, Issledovaniye svobodnykh kolebaniy gofrirovannykh tsilindricheskikh obolochek, Teoret. i prikl. Mekhanika, 47, No.1, (2010) $106-113$.

9. N. P. Semenyuk, I. Yu. Babich, N. B. Zhukova, Natural Vibrations of Corrugated Cylindrical Shells, Int. Appl. Mech., 41, No.5 (2005) $512-519$.

10. Werner Soedel, Vibrations of Shells and Plates, 3rd Ed., Rev. and Expanded. -Marcel Dekker, Inc., NY 2004. - 592 p.

11. Zeng He, Guixiang Liu, Wen Jiang, Zheng Huang, Dongliang Zhang, Nonlinear Dynamic Responses of a Corrugated Shell Structure under Uniform Load, J. of Eng. Mech., 140, Issue 6 (2014). Doi: org/10.1061/(ASCE)EM.1943-7889.0000717. 